\title{
Silencing of the Expression of the Immunoglobulin Kappa Gene in Non-B Cells
}

\author{
JACQUELINE W. PIERCE, ${ }^{1}+*$ ANN M. GIFFORD, ${ }^{1}$ AND DAVID BALTIMORE ${ }^{2}$ \\ Whitehead Institute for Biomedical Research, Cambridge, Massachusetts $02142,{ }^{1}$ and \\ The Rockefeller University, 1230 York Avenue, New York, New York 10021 2
}

Received 9 October 1990/Accepted 10 December 1990

\begin{abstract}
Although the activating factor NF-kB can be present in the nucleus of many cell types, transcription and rearrangement of the immunoglobulin kappa chain gene is restricted to cells of the $B$ lineage. Part of this specificity is determined by sequences within the major intron of the kappa gene that specifically silence gene expression in non-B cells ( $T$ cells and HeLa cells). These sequences are found in a 232-bp fragment located 5' of the NF-kB binding sequence of the enhancer. When this fragment is added back upstream of an active NF- $\kappa B$ site, it specifically decreases the expression of a linked gene by more than 10-fold in activated $T$ cells but it has no effect on expression in B cells. The kappa silencer region acts in an orientation- and distanceindependent manner and appears to be composed of multiple negative elements. The kappa silencer may act to restrict transcription and rearrangement of the $C_{k}$ locus to cells of the $B$ lineage.
\end{abstract}

The immunoglobulin kappa chain gene is under precise developmental control. This gene is expressed only in cells of the B-lymphocyte lineage. The transcription of the murine kappa locus is regulated by the binding of nuclear factors to DNA sequences within the $V_{k}$ promoters and the two enhancer regions, one located within the $J-C_{k}$ intron and another located downstream of the $C_{\kappa}$ coding region $(5,13$, $15,32,40,42)$. Several regulatory sequences within the intron enhancer are important for enhancer function in $\mathrm{B}$ cells $(14,27,46)$. These include the $E$ motifs ( $\kappa E 1, \kappa E 2$, and $\kappa E 3)$, which are homologous to the protein-binding consensus sequence CAGGTGGC in the immunoglobulin heavychain enhancer and the $\mathrm{kB}$ site, GGGACTTTCC, which binds the nuclear factor NF-кB (46). The activation of both $C_{k}$ transcription and rearrangement occurs at the pre-B- to $B$-cell transition and is correlated with the induction of

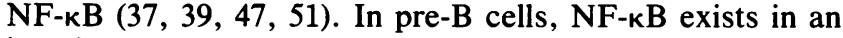
inactive cytoplasmic form where it is complexed with an inhibitor, IкB (3). Upon induction of pre-B cells, IкB undergoes a phosphorylation event and NF- $\mathrm{BB}$ is translocated to the nucleus, where it can bind DNA $(2-4,17,47)$. The DNA-binding form of NF- $\mathrm{kB}$ is present constitutively only in mature $B$ cells and plasma cells which express kappa light-chain genes. Binding of NF-kB to the enhancer is crucial to the activation of gene transcription, since mutation of the NF-kB binding site abolishes kappa enhancer activity (27).

The NF-kB protein was originally identified in cells of the $B$ lineage. However, NF-kB and related factors can be induced in many cell types by treatment with phorbol 12-myristate 13-acetate (PMA), interleukin-1, doublestranded RNA, or viral infection $(29,47,52)$. Treatment of $T$ cells or HeLa cells with PMA induces NF-kB $(2,47)$. In non-B cells, NF-кB and related proteins have pleiotropic effects, activating a broad range of different genes (reviewed in reference 28). For example, NF-kB is induced upon T-cell activation, and it stimulates transcription of many genes,

\footnotetext{
* Corresponding author.

$\dagger$ Present address: Rosenstiel Basic Medical Sciences Research Center, Brandeis University, Waltham, MA 02254-9110.
}

including interleukin-2 receptor alpha chain (7). Significantly, NF- $\mathrm{kB}$ can also activate several viral enhancers, including the human immunodeficiency virus enhancer, the simian virus 40 enhancer, and the cytomegalovirus enhancer $(8,33)$.

Since NF- $\mathrm{B}$ can stimulate so many different genes in $\mathrm{T}$ and $B$ cells, it is clear that NF- $\mathrm{BB}$ by itself cannot account for the precise tissue-specific regulation of each of these genes. There must be additional regulatory elements which modify the activity of NF- $\mathrm{NB}$ and prevent the inappropriate activation of all these genes at once. We and others have previously observed that the NF- $\mathrm{B}$ binding sequence alone can activate transcription in either $B$ cells or non-B cells treated with PMA $(36,41,56)$. However, a 476-bp AluI fragment containing the kappa intron enhancer was not active in T cells treated with PMA (41). Here we present evidence that sequences associated with the kappa chain intron enhancer actively silence gene expression in non- $B$ cells. The role of the kappa silencer may be to restrict activation of the kappa gene to cells of the $B$ lineage.

\section{MATERIALS AND METHODS}

Cell lines, transfections, and enzymatic assays. Jurkat (human T cell), EL4 (mouse T cell), YAC-1 (mouse T cell), and S194 (mouse B-lineage plasma cell) cell lines were grown in RPMI 1640 medium with $10 \%$ fetal bovine serum. HeLa cells were grown in Dulbecco's modified Eagle's medium (DME) with $10 \%$ bovine calf serum. Cells were transfected by the DEAE-dextran technique as modified by Grosschedl and Baltimore (21) with $10 \mu \mathrm{g}$ of test plasmid for $10^{7}$ cells. The transfected T or HeLa cells were grown in two flasks, and at $24 \mathrm{~h}$ after transfection, one half of the cells were treated with PMA $(50 \mathrm{ng} / \mathrm{ml}$; Sigma) for $18 \mathrm{~h}$. The Jurkat cells were induced with both PMA $(50 \mathrm{ng} / \mathrm{ml})$ and $2 \mu \mathrm{g}$ of phytohemagglutinin (PHA; ICN Immunobiologicals, Lisle, Ill.) per ml. Cells were harvested at $42 \mathrm{~h}$ after transfection, and extracts were prepared and heat treated for $7 \mathrm{~min}$ at $60^{\circ} \mathrm{C}$ as described previously (41). Chloramphenicol acetyltransferase (CAT) assays were carried out with $50 \mu \mathrm{g}$ of protein (Jurkat, EL4, YAC-1, and HeLa cells) or $20 \mu \mathrm{g}$ of protein (S194 cells) as described previously $(20,41)$. Each result represents the 


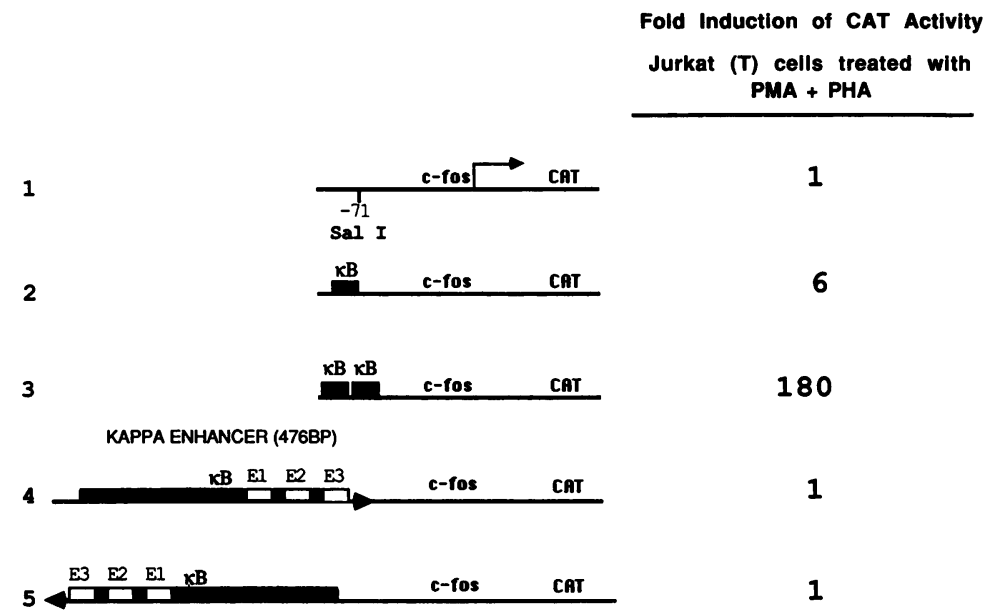

FIG. 1. Immunoglobulin kappa intron enhancer is not inducible in T cells. Relative levels of CAT activity from plasmids transfected into Jurkat cells. After $24 \mathrm{~h}$, one half of the cells were treated with PMA $(50 \mathrm{ng} / \mathrm{ml})$ and PHA $(2 \mu \mathrm{g} / \mathrm{ml})$ for $18 \mathrm{~h}$. Values for fold induction indicate percent conversion in PMA-induced cells divided by percent conversion in uninduced cells. Each value represents the average of three or more independent transfections, and the standard deviation was less than $20 \%$. The thin arrow represents the start of transcription. Solid boxes represent sequences which bind NF-kB within a 26-bp oligonucleotide or the kappa enhancer 476-bp AluI fragment. Line 1, $\Delta 71-f o s$-CAT plasmid (18); lines 2 and 3, described in Pierce et al. (41); lines 4 and 5, described in Lenardo et al. (27).

average of three or more independent transfections. The standard deviation was less than $20 \%$.

Plasmid constructions. The plasmid $\Delta 71$-fos-CAT was described by Gilman et al. (18) and contains a minimal c-fos promoter $(-71$ to +109$)$ driving the cat gene. The sequence upstream of the c-fos promoter is from the pUC13 polylinker, including a unique HindIII site and SalI site. Plasmids

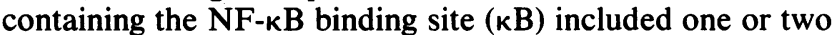
copies of the 26-bp oligonucleotide sequence TCGACA GAGGGGACTTTCCGAGAGGC inserted at the unique Sall site of $\Delta 71$-fos-CAT as described by Pierce et al. (41) (for the $\mathrm{J} 17$ plasmid, see line 2 of Fig. 2 in reference 41 ; for J18 see line 5 of Fig. 2 in reference 41). The Kappa-CAT plasmid contains the 476-bp AluI fragment (positions 3692 to 4166, numbering according to Emorine et al. [13]) from the kappa intron inserted at the SalI site in $\Delta 71$-fos-CAT as described by Lenardo et al. (27). For constructs J44, J45, J53, J58, J59, J61, J65, and J56 in Fig. 3, test fragments were inserted at a unique $S a l$ site immediately upstream of the $к B$ site in the plasmid $\mathrm{J} 17$, which contains one $\kappa \mathrm{B}$ site driving transcription of the c-fos-cat gene. The plasmids in Fig. 4 were derived by inserting test fragments at a unique HindIII site $18 \mathrm{bp}$ upstream of the two $\mathrm{kB}$ sites in $\mathrm{J} 18$.

\section{RESULTS}

Restricted activity of the kappa enhancer in $T$ cells. The kappa intron enhancer is known to be B-cell specific $(40,42)$. Its activity is dependent on the binding of nuclear factor NF- $k B$, which is induced during maturation of pre-B cells. Because NF-kB and related factors can also be induced in $T$ and HeLa cells treated with PMA, we tested the activity of the kappa enhancer in these cells. We inserted the 476-bp AluI fragment containing the mouse kappa intron enhancer into a test plasmid containing the mouse c-fos promoter driving the cat gene (Fig. 1; line 1, test plasmid; lines 4 and 5 , with kappa enhancer). In addition, we tested plasmids containing either one or two copies of the binding sequence for NF- $\mathrm{NB}(\kappa \mathrm{B})$ inserted as a 26-bp oligonucleotide immediately upstream of the promoter (Fig. 1, lines 2 and 3). These plasmids were transfected into $T$ cells (Jurkat), and after 24 $h$, half of the cells were treated with PMA and PHA to induce NF-кB. We determined CAT enzyme activity after 42 $h$ as a measure of gene transcription.

Consistent with previous observations, the plasmids containing one $\kappa \mathrm{B}$ site gave 6-fold induction while those containing two $\kappa B$ sites gave 180 -fold induction of CAT activity after treatment with PMA and PHA (41). The kappa enhancer fragment, by contrast, showed no induction under these conditions even though its activity in B cells is equivalent to that of two $\mathrm{kB}$ sites (41). A similar lack of activity was obtained with other T-cell lines (EL4 and YAC-1) and with Jurkat cells induced with PMA alone. Thus, the kappa enhancer appears to have an activity that restricts its action to $\mathrm{B}$ cells.

To determine whether particular sequences within the enhancer region act as negative elements in $T$ cells, we inserted either the kappa enhancer fragment (476 bp) or a nonspecific spacer fragment from pUC19 (473 bp) into the plasmid containing two $\mathrm{kB}$ sites. These fragments of DNA were placed between the $\kappa \mathrm{B}$ sites and the promoter (Fig. 2). The pUC19 spacer fragment had only a small effect on the ability of $\kappa B$ sites to drive transcription of the cat gene, but the same size fragment from the kappa enhancer reduced activity to $9 \%$ of the control. This result implies that the kappa enhancer region contains sequences which dominantly inhibit transcription in activated T cells. We will call these sequences silencers for the reasons given below.

Silencing sequences. To search for silencer elements within the kappa enhancer region, the whole enhancer or fragments from the 476-bp enhancer region were added back at a unique $S a l I$ site upstream of the $\kappa B$ site in the $\kappa B$-fos-CAT test plasmids (Fig. 3A and B). In these "add back" experiments, the position of the $\kappa \mathrm{B}$ site, fos promoter, and cat gene remained constant. These plasmids were then transfected into Jurkat cells, and one half of the cells were treated with PMA and PHA to induce NF- $\mathrm{BB}$. Under these conditions, a single $\kappa B$ site gives an average of sixfold induction of CAT activity (Fig. 1, line 2). When the entire 476-bp kappa fragment was present upstream in either orientation, the induced CAT activity was decreased to about $30 \%$ of that seen with the $\kappa \mathrm{B}$ site alone (Fig. 3B, J45 and J44 versus J17). 


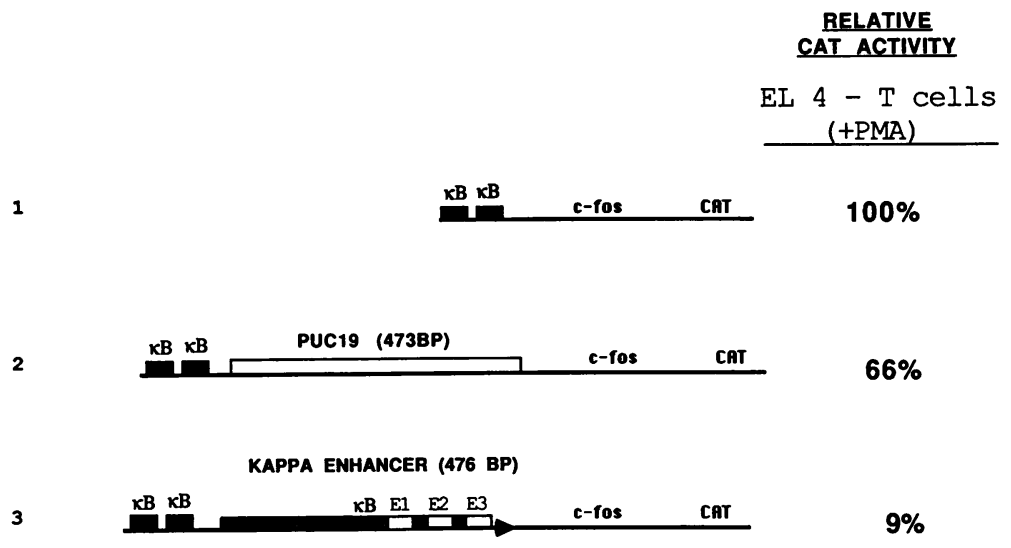

FIG. 2. Sequences within the kappa enhancer 476-bp fragment decrease gene expression in T cells. Plasmids were transfected into EL4 $\mathrm{T}$ cells, and after $24 \mathrm{~h}$ cells were treated with PMA for $18 \mathrm{~h}$ as described in Materials and Methods. Relative CAT values were obtained by comparison with activity from the plasmid shown in line 1 . The solid boxes indicate NF-kB binding sequences within a 26-bp oligonucleotide or within the kappa enhancer.

In S194 myeloma cells, plasmids containing the kappa 476-bp fragment gave five times more activity than the KB-only constructs (Fig. 3B). Thus, in the presence of two $\kappa \mathrm{B}$ sites, the whole enhancer has the predicted positive effect in B cells but a net negative effect in T cells. This result highlights the power of the negative elements in $\mathrm{T}$ cells.

We then attempted to separate the positive and negative elements by dividing the enhancer region in half so that the $\kappa B$ site and the three $E$ motifs were in the $3^{\prime}$ half and the remaining 232 bp were in the $5^{\prime}$ half. This was done by inserting a unique $B$ gllI site at position 3924 , just upstream of the NF-kB site (Fig. 3A). Some positive elements may still be present in the $5^{\prime}$ half $(232 \mathrm{bp})$ of the enhancer $(34,43)$. The $B g l I I$ site had no effect on kappa enhancer function in B or T cells (data not shown). In either orientation, the 232-bp fragment from the $5^{\prime}$ half (positions 3692 to 3924) decreased transcription in $\mathrm{T}$ cells about 10 -fold relative to the $\mathrm{\kappa B}$-foscat plasmid alone (Fig. 3B, J53 and J58 versus $\mathrm{J} 17$ ). When the same plasmids were tested in B cells, the 232-bp fragment did not have any effect, suggesting that it contains mainly negative regulators whose activity is specific to non-B cells.

We also tested the $3^{\prime}$ half of the kappa enhancer region (3924 to 4166) for its effect on transcription. This fragment contains the $\kappa \mathrm{B}, \mathrm{E} 1, \mathrm{E} 2$, and E3 sites. It increased trànscription about twofold in $\mathrm{T}$ cells but had a much greater effect in B cells (Fig. 3B, J59). This difference may reflect the fact that $E$ motifs preferentially enhance transcription in $B$ cells, but it is also possible that negative elements exist within the $3^{\prime}$ half of the kappa enhancer. Further study of this issue is required.

To determine whether the effect of the $5^{\prime}$ silencer region was specific to $T$ cells or could be observed in nonlymphoid cells as well, we tested our constructs in $\mathrm{HeLa}$ cells treated with PMA to induce NF-кB. Plasmids containing the 232-bp fragment showed a silencing effect, giving three- to fivefold less activity than those containing $\kappa \mathrm{B}$ alone (Fig. $3 \mathrm{~B}$ ). These results suggest that sequences in the kappa enhancer region can decrease gene expression in nonlymphoid cells. We also tested whether the intact kappa enhancer could be induced in HeLa cells upon treatment with PMA. The Kappa-CAT plasmid (Fig. 1, line 5) was tested in HeLa cells, and the 476-bp kappa enhancer fragment gave little or no enhancer activity in PMA-induced HeLa cells, while constructs con- taining just one NF- $\mathrm{BB}$ site gave 8- to 14-fold induction of gene expression (data not shown). The silencing in HeLa cells was not as great as that in T-cell lines, perhaps due to real differences in silencer function in these cell types but alternatively because of differences in transfection efficiency. In HeLa cells, the number of plasmid molecules per cell may be greater and the putative proteins binding to the negative elements could be limiting.

The kappa silencer was also tested for the ability to act on heterologous enhancers to determine whether this silencer was specific for $\kappa \mathrm{B}$-containing enhancers. We found no evidence for silencing of the $T$ cell receptor $\alpha$, Rous sarcoma virus, or Moloney murine leukemia virus enhancers (data not shown), suggesting that the silencing may be specific for enhancers containing $\kappa \mathrm{B}$ sites.

Location of negative elements. To dissect the negative elements in the kappa enhancer in more detail, we constructed a panel of BAL-31 deletions within the 232-bp fragment $5^{\prime}$ half and tested these smaller subfragments for their ability to silence gene activation. When the entire 232-bp fragment was tested in two different $T$-cell lines (Jurkat and YAC-1), this region gave about 10 -fold silencing of gene activity (Fig. 3C, J58). A 174-bp fragment (positions 3750 to 3924 ) gave full silencing activity, but a 58-bp fragment (3866 to 3924) showed less than fivefold silencing (Fig. $3 \mathrm{C}, \mathrm{J} 61$ and J65). Thus, deletion of the region from 3750 to 3866 resulted in the loss of some but not all silencing activity. Deletion of 3866 to 3888 further reduced the level of silencing (J56). Similar results were obtained in both Jurkat and YAC-1 T-cell lines. This suggests that at least two regions within the 232-bp fragment are important for negative regulation.

Silencer elements, originally defined in Saccharomyces cerevisiae, have the property of acting at a distance to negatively regulate transcription (9). To test whether the negative elements in the 232 -bp region could act at a distance, we inserted this fragment either 18 or $250 \mathrm{bp}$ upstream of the promoter in a test plasmid containing two $\mathrm{\kappa B}$ sites (Fig. 4; J18, test plasmid, J87 [18 bp upstream], and J110 [250 bp upstream]). In either position, the 232-bp fragment gave about 10-fold silencing in $T$ cells while no effect was seen in $B$ cells. Thus, this region may be defined as a silencer because its function is distance and orientation independent. 


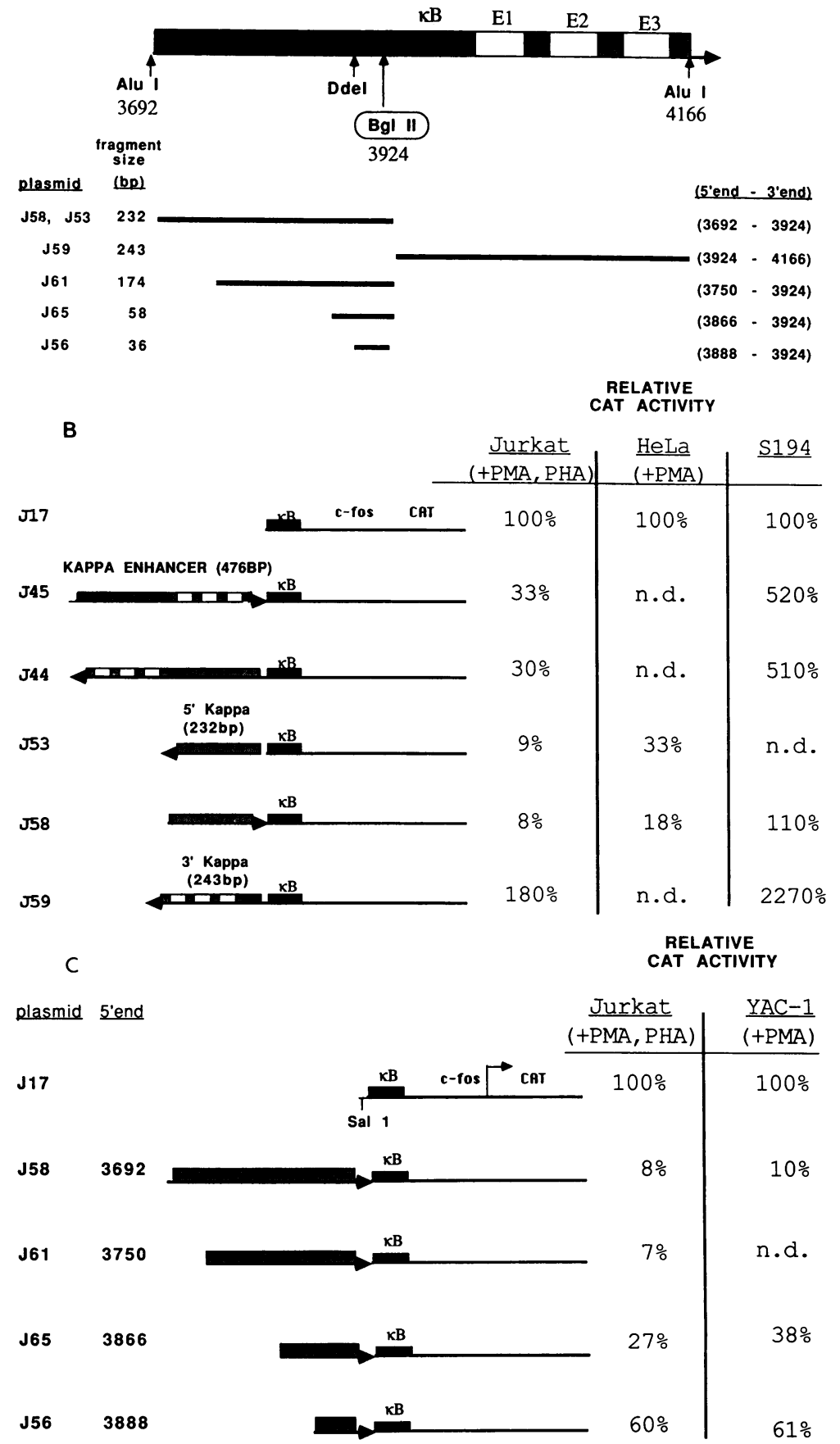

FIG. 3. Location of negative regulatory elements. (A) Kappa intron enhancer and subfragments. Numbering of the 476-bp AluI fragment from the J-C $\mathrm{C}_{\mathrm{k}}$ intron is according to Emorine et al. (13). Locations of relevant restriction sites within the $5^{\prime}$ half are shown. A unique BglII site (circled) was introduced at position 3924 by site-directed mutagenesis. Subfragments to be tested for their effects on transcription are shown below, with the names of the test plasmids used in panels B and C. (B) Effect of kappa enhancer or subfragments on transcription was measured by inserting fragments upstream of the NF-кB site at a unique SalI site in test plasmid J17 (top line). Plasmids were transfected into the cell lines indicated, and T cells (Jurkat) and HeLa cells were treated to induce NF- $\mathrm{kB}$. The relative levels of CAT activity after induction are given. Values were obtained by comparison with that from the plasmid J17 (top line). n.d., Not done. (C) A panel of BAL-31 deletion fragments were made within the 232-bp AluI-BglII fragment (3692 to 3924) as shown in panel A. The 5' endpoints of the fragments are indicated. Each fragment extended to the BglII site at 3924 ( $3^{\prime}$ end). Fragments were tested for their effect on transcription by inserting each one at the SalI site upstream of the $\mathrm{\kappa B}$ site in plasmid J17. Relative CAT activity after induction of Jurkat and YAC-1 T cells was obtained by comparison with that from J17 (top line). 


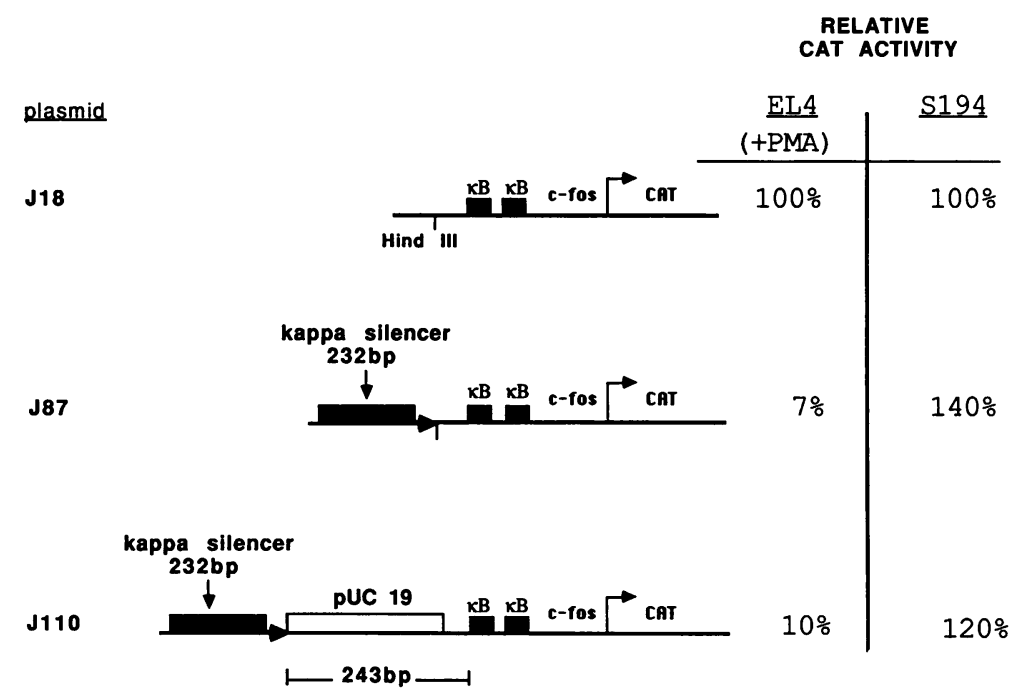

FIG. 4. Position independence of silencer function. The 232-bp AluI-Bg/II fragment was tested for effects on transcription by insertion at different positions in the test plasmid $\mathrm{J} 18$. Line $1, \mathrm{~J} 18$, no silencer. Line 2, J87, insertion at the HindIII site $18 \mathrm{bp}$ upstream of the $\mathrm{kB}$ sites. Line 3, J110, insertion 243 bp from the $\mathrm{kB}$ sites (DNA sequence between the silencer fragment and the $\mathrm{\kappa B}$ site is from pUC19 XmnI-SspI fragment and pUC13 polylinker sequnces). Relative CAT activities in T cells (EL4) and B cells (S194) were obtained by comparison with that from J18. The J18 plasmid containing two $\mathrm{KB}$ sites was used to obtain higher absolute levels of CAT activity.

\section{DISCUSSION}

We found that tissue-specific expression of the immunoglobulin kappa intron enhancer is the result of both positive and negative regulation. The kappa enhancer contains negative elements which act to silence gene expression in non-B-cell types, specifically $\mathrm{T}$ cells and HeLa cells. The silencer region is located within a 232-bp fragment upstream of the $\kappa B$ site. This region can function in an orientation- and distance-independent fashion. The silencer region is probably important in restricting kappa enhancer activity to B cells and may act to prevent the inappropriate activation of the kappa gene by NF-кB induced in other cell types. Thus, activation of the kappa gene in developing $B$ cells may be a two-step process, involving both release of the kappa silencer and induction of NF-kB.

The kappa silencer region is within the $5^{\prime}$ half of the previously described intron enhancer and appears to be composed of multiple negative elements. The negative elements do not appear to overlap with the $\kappa B$ site, as has been described for negative elements in the human beta interferon gene (19). The composite nature of the kappa silencer is similar to the silencer which has recently been identified upstream of the T-cell receptor alpha chain (TCR $\alpha)$ gene enhancer (54). As with the TCR $\alpha$ silencer, more than one sequence element is necessary for the full silencing effect. In addition, the immunoglobulin heavy-chain enhancer has been reported to be associated with multiple negative elements $(25,26,53)$. It is likely that silencers, like enhancers, may be composed of multiple sequence motifs.

The mechanism of silencer function is not known. It is possible that DNA sequences within the silencer region may act as binding sites for nuclear factors which act as repressors of transcription. We have identified one A+T-rich sequence which binds protein (data not shown). This site (ATTAATTTAT, positions 3825 to 3834 ) is located with a region that is important for full silencing activity. Currie and Roeder have detected protein binding to the same A+T-rich sequence, and they suggested that OCT-2 can bind to this site as well as to two other sites in this region of the mouse kappa enhancer $(12,50)$. An octamer-binding site has also been identified in the human kappa enhancer (35). It is possible that an octamer-binding protein (i.e., OCT-1) could act to negatively regulate transcription in non-B cells. Interestingly, a negative regulatory function has been proposed for another protein that binds the octamer sequence, the OCT-3 (also known as NF-A3) protein in teratocarcinoma cells (30). This factor binds both the octamer ATTTGCAT and the A+T-rich sequence TTAAAATCA (38). In addition, octamer sequences have been implicated in negative regulation of the immunoglobulin heavy-chain enhancer in $\mathrm{T}$ cells (57). The specific release of silencing in B cells might reflect competition by the B-cell-specific protein OCT-2. However, silencing was also observed in EL4 T cells, which possess significant levels of OCT-2. Thus, any regulation involving octamer-binding factors is likely to be complex.

Additional proteins which bind within this region of the kappa enhancer have been observed $(12,23,24)$. Currie and Roeder have identified a nuclear factor, NF-kE*, which was found to be more abundant in HeLa cells than in B cells (12). Our studies show that deletion of the $\mathrm{\kappa E}^{*}$ binding region (positions 3866 to 3888 ) gives about twofold less silencing (Fig. 3C). Thus, NF-kE* may contribute to silencer function. The preferential distribution of this protein in non-B cells is consistent with the possibility that this protein could act as a tissue-specific repressor.

An alternative mechanism by which silencer sequences may act to decrease gene transcription is through interaction with the nuclear matrix. In the yeast mating type loci, the silencer E site binds a protein, RAP-1, which appears to be necessary for formation of DNA loops in a nuclear scaffold assay $(22,48,49)$. The sequences flanking both the heavychain and kappa enhancers are $A+T$ rich and have been suggested to act as matrix attachment regions (MARs) and potential binding sites for topoisomerase II $(10,11)$. In the case of the kappa enhancer, the previously reported MARs lie outside the 476-bp AluI fragment and thus are not present in the silencer region. These adjacent MARs in the kappa intron have not been tested for possible function as negative 
regulatory elements. However, the 232-bp silencer region is highly $\mathrm{A}+\mathrm{T}$ rich and includes a protein-binding sequence, ATTAATTTAT, which has some homology to the consensus sequence for topoisomerase II binding, GTNWAYATT NATNNR (12, 16; data not shown). In the immunoglobulin heavy-chain enhancer, A+T-rich elements are found within the negative regulatory regions, and the silencers adjacent to the TCR $\alpha$ chain enhancer also include $\mathrm{A}+\mathrm{T}$-rich regions with homology to the topoisomerase II consensus $(25,26$, $44,53,54)$. It is not yet clear whether topoisomerase II binding is involved in silencer function. It is likely that silencing involves additional elements specific to each silencer, because topoisomerase II alone could not account for the different patterns of tissue specificity observed for each silencer.

The kappa silencer may play a role in regulating both transcription and rearrangement of the $C_{k}$ locus. Transcription of the unrearranged $C_{k}$ gene is known to be correlated with kappa rearrangement $(37,39,51)$. In fact, treatment of pre-B cells with lipopolysaccharide activates not only NF- $\mathrm{B}$ and kappa transcription but also kappa gene rearrangement (45). Germ line transcription of the immunoglobulin heavy-chain genes has been correlated with recombination of these genes, and it has been proposed that transcription of the germ line locus is a prerequisite for recombination $(1,6)$. Activation of $N F-\kappa B$ in $T$ cells might lead to transcription and rearrangement of the $C_{k}$ locus, since the recombinational apparatus appears to be the same for B and T cells. Thus, the kappa silencer may be important in restricting both germ line $C_{k}$ transcription and kappa rearrangement to $B$ cells. A similar role for silencer elements in regulation of gene rearrangement has been proposed for the TCR $\alpha$ locus (54). Silencers associated with the TCR $\alpha$ enhancer may act to prevent $J \alpha$ segments from rearranging in $\gamma \delta \mathrm{T}$-cell lines, thus regulating the differentiation of $\alpha \beta$ versus $\gamma \delta \mathrm{T}$-cell lineages. The development of cells along the B lineage may involve specific release from kappa silencer activity. We expect that silencers will be as important as enhancers in dictating the tissue-specific patterns of gene expression.

\section{ACKNOWLEDGMENTS}

We thank Astar Winoto, Mark S. Schlissel, and Ranjan Sen for helpful comments on the manuscript.

This work was funded by a Special Fellowship of the Leukemia Society of America to J.W.P., Public Health Service grant GM39458 to D.B., and Public Health Service grant GM-43874-01 from the National Institutes of Health to Ranjan Sen.

\section{REFERENCES}

1. Alt, F., T. Blackwell, and G. Yancopoulos. 1987. Development of the primary antibody repertoire. Science 238:1079-1087.

2. Baeuerle, P. A., and D. Baltimore. 1988. Activation of DNAbinding activity in an apparently cytoplasmic precursor of the NF-KB transcription factor. Cell 53:211-217.

3. Baeuerle, P. A., and D. Baltimore. 1988. IאB: a specific inhibitor of the NF-KB transcription factor. Science 242:540-546.

4. Baeuerle, P. A., M. Lenardo, J. W. Pierce, and D. Baltimore. 1988. Phorbol ester-induced activation of the NF- $\mathrm{kB}$ transcription factor involves dissociation of an apparently cytoplasmic NF-kB/inhibitor complex. Cold Spring Harbor Symp. Quant. Biol. 53:789-798.

5. Bergman, Y., D. Rice, R. Grosschedl, and D. Baltimore. 1984. Two regulatory elements for $\kappa$ immunoglobulin gene expression. Proc. Natl. Acad. Sci. USA 81:7041-7045.

6. Blackwell, T., M. Moore, G. Yancopoulos, H. Suh, S. Lutzker, E. Selsing, and F. Alt. 1986. Recombination between immunoglobulin variable region gene segments is enhanced by transcrip- tion. Nature (London) 324:585-589.

7. Bohnlein, E., J. W. Lowenthal, M. Siekevitz, D. W. Ballard, B. R. Franza, and W. C. Greene. 1988. The same inducible protein regulates mitogen activation of both the interleukin-2 receptor alpha gene and type 1 HIV. Cell 53:827-836.

8. Boshart, M., F. Weber, G. Jahn, K. Dorsch-Hasler, B. Fleckenstein, and W. Schaffiner. 1985. A very strong enhancer is located upstream of an immediate early gene of human cytomegalovirus. Cell 41:521-530.

9. Brand, A. H., L. Breeden, J. Abraham, R. Sternglanz, and K. Nasmyth. 1985. Characterization of a silencer in yeast: a DNA sequence with properties opposite to those of a transcriptional enhancer. Cell 41:41-48.

10. Cockerill, P. N., and W. T. Garrard. 1986. Chromosomal loop anchorage of the kappa immunoglobulin gene occurs next to the enhancer in a region containing topoisomerase II sites. Cell 44:273-282.

11. Cockerill, P. N., M. H. Yuen, and W. T. Garrard. 1987. The enhancer of the immunoglobulin heavy chain locus is flanked by presumptive chromosomal loop anchorage elements. J. Biol. Chem. 262:5394-5397.

12. Currie, R. A., and R. G. Roeder. 1989. Identification of an octamer-binding site in the mouse kappa light-chain immunoglobulin enhancer. Mol. Cell. Biol. 9:4239-4247.

13. Emorine, L., M. Kuehl, L. Weir, P. Leder, and E. E. Max. 1983. A conserved sequence on the immunoglogulin $J_{k}-C_{k}$ intron: possible enhancer element. Nature (London) 304:447-449.

14. Ephrussi, A., G. M. Church, S. Tonegawa, and W. Gilbert. 1985. B-lineage specific interactions of an immunoglobulin enhancer with cellular factors in vivo. Science 227:134-140.

15. Falkner, F. G., and H. G. Zachau. 1984. Tissue specificity of the initiation of immunoglobulin $\kappa$ gene transcription. Nature (London) 310:71-74.

16. Gasser, S. M., T. Laroche, J. Falquiet, E. Boy de la Tour, and U. K. Laemmli. 1985. Metaphase chromosome structure: involvement of topoisomerase II. J. Mol. Biol. 188:613-629.

17. Ghosh, S., and D. Baltimore. 1990. Activation in vitro of NF-kB by phosphorylation of its inhibitor, IKB. Nature (London) 344:678-682.

18. Gilman, M. Z., R. N. Wilson, and R. A. Weinberg. 1986. Multiple protein-binding sites in the 5'-flanking region regulate c-fos expression. Mol. Cell. Biol. 6:4305-4316.

19. Goodbourn, S., and T. Maniatis. 1988. Overlapping positive and negative regulatory domains of the human $\beta$-interferon gene. Proc. Natl. Acad. Sci. USA 85:1447-1451.

20. Gorman, C. M., L. F. Moffat, and B. H. Howard. 1982. Recombinant genomes which express chloramphenicol acetyltransferase in mammalian cells. Mol. Cell. Biol. 2:1044-1051.

21. Grosschedl, R., and D. Baltimore. 1985. Cell type specificity of immunoglobulin gene expression is regulated by at least 3 DNA sequence elements. Cell 41:885-897.

22. Hofmann, J. F.-X., T. Laroche, A. H. Brand, and S. M. Gasser. 1989. RAP-1 factor is necessary for loop formation in vitro at the silent mating type locus $H M L$. Cell 57:725-737.

23. Hromas, R., U. Pauli, A. Marcuzzi, D. Lafrenz, H. Nick, J. Stein, G. Stein, and B. Van Ness. 1988. Inducible DNA-protein interactions of the murine kappa immunoglobulin enhancer in intact cells: comparison with in vitro interactions. Nucleic Acids Res. 16:953-967.

24. Hromas, R., and B. Van Ness. 1986. Nuclear factors bind to regulatory regions of the mouse kappa immunoglobulin gene. Nucleic Acids Res. 14:4837-4848.

25. Imler, J.-L., C. Lemaire, C. Wasylyk, and B. Wasylyk. 1987. Negative regulation contributes to tissue specificity of the immunoglobulin heavy-chain enhancer. Mol. Cell. Biol. 7:25582567.

26. Kadesch, T., P. Zerros, and D. Ruesinsky. 1986. Functional analyses of the murine IgH enhancer: evidience for a negative control of cell type specificity. Nucleic Acids Res. 14:82098221.

27. Lenardo, M., J. W. Pierce, and D. Baltimore. 1987. Proteinbinding sites in Ig gene enhancers determine transcriptional activity and inducibility. Science 236:1573-1577. 
28. Lenardo, M. J., and D. Baltimore. 1989. NF-кB: a pleiotropic mediator of inducible and tissue-specific gene control. Cell 58:227-229.

29. Lenardo, M. J., C.-M. Fan, T. Maniatis, and D. Baltimore. 1989. The involvement of NF-kB in $\beta$-interferon gene regulation reveals its role as widely inducible mediator of signal transduction. Cell 57:287-294.

30. Lenardo, M. J., L. Staudt, P. Robbins, A. Kuang, R. C. Mulligan, and D. Baltimore. 1989. Repression of the IgH enhancer in teratocarcinoma cells associated with a novel octamer factor. Science 243:544-546.

31. Libermann, T. A., and D. Baltimore. 1990. Activation of interleukin- 6 gene expression through the NF-кB transcription factor. Mol. Cell. Biol. 10:2327-2334.

32. Meyer, K. B., and M. S. Neuberger. 1989. The immunoglobulin $\kappa$ locus contains a second, stronger B-cell specific enhancer which is located downstream of the constant region. EMBO J. 8:1959-1964.

33. Nabel, G., and D. Baltimore. 1987. An inducible transcription factor activates expression of human immunodeficiency virus in T cells. Nature (London) 326:711-713.

34. Nelms, K., R. Hromas, and B. Van Ness. 1990. Identification of a second inducible DNA-protein interaction in the kappa immunoglobulin enhancer. Nucleic Acids Res. 18:1037-1043.

35. Nelms, K., and B. Van Ness. 1990. Identification of an octamer binding site in the human kappa light-chain enhancer. Mol. Cell. Biol. 10:3843-3846.

36. Nelsen, B., L. Hellman, and R. Sen. 1988. The NF-kB binding site mediates phorbol ester-inducible transcription in nonlymphoid cells. Mol. Cell. Biol. 8:3526-3531.

37. Nelson, K. J., D. E. Kelley, and R. P. Perry. 1985. Inducible transcription of the unrearranged $\kappa$ constant region is a common feature of pre-B cells and does not require DNA or protein synthesis. Proc. Natl. Acad. Sci. USA 82:5305-5309.

38. Okamoto, K., H. Okazawa, A. Okuda, M. Sakai, M. Muramatsu, and H. Hamada. 1990. A novel octamer binding transcription factor is differentially expressed in mouse embryonic cells. Cell 60:461-472.

39. Perry, R. P., D. E. Kelley, C. Coleclough, J. G. Seidman, P. Leder, S. Tonegawa, G. Matthyssens, and M. Weigert. 1980. Transcription of mouse $\kappa$-chain genes: implications for allelic exclusion. Proc. Natl. Acad. Sci. USA 77:1937-1941.

40. Picard, D., and W. Schaffner. 1984. A lymphocyte-specific enhancer in the mouse immunoglobulin $\kappa$ gene. Nature (London) 307:80-82.

41. Pierce, J. W., M. Lenardo, and D. Baltimore. 1988. Oligonucleotide that binds nuclear factor NF-kB acts as a lymphoidspecific and inducible enhancer element. Proc. Natl. Acad. Sci. USA 85:1482-1486.

42. Queen, C., and D. Baltimore. 1983. Immunoglobulin gene transcription is activated by downstream sequence elements. Cell
33:741-748.

43. Queen, C., and J. Stafford. 1984. Fine mapping of an immunoglobulin gene activator. Mol. Cell. Biol. 4:1042-1049.

44. Scheuermann, R. H., and U. Chen. 1989. A developmentalspecific factor binds to suppressor sites flanking the immunoglobulin heavy-chain enhancer. Genes Dev. 3:1255-1266.

45. Schlissel, M. S., and D. Baltimore. 1989. Activation of immunoglobulin kappa gene rearrangement with induction of germline kappa gene transcription. Cell 58:1001-1007.

46. Sen, R., and D. Baltimore. 1986. Multiple nuclear factors interact with the immunoglobulin enhancer sequences. Cell 46:705-716.

47. Sen, R., and D. Baltimore. 1986. Inducibility of $k$ immunoglobulin enhancer binding protein $\mathrm{NF}-\mathrm{KB}$ by a posttranslational mechanism. Cell 47:921-928.

48. Shore, D., and K. Nasmyth. 1987. Purification and cloning of a DNA binding protein from yeast that binds to both silencer and activator elements. Cell 51:721-732.

49. Shore, D., D. J. Stillman, A. H. Brand, and K. A. Nasmyth. 1987. Identification of silencer binding proteins from yeast: possible roles in SIR control and DNA replication. EMBO J. 6:461-467.

50. Staudt, L. M., R. G. Clerc, H. Singh, J. H. LeBowitz, P. A. Sharp, and D. Baltimore. 1988. Cloning of a lymphoid-specific cDNA encoding a protein binding the regulatory octamer DNA motif. Science 241:577-580.

51. Van Ness, B. G., M. Weigert, C. Coleclough, E. L. Mather, D. E. Kelley, and R. P. Perry. 1981. Transcription of the unrearranged mouse $C_{k}$ locus: the initiation region and comparison of activity with a rearranged $V_{k}-C_{k}$ gene. Cell 27:593-602.

52. Visvanathan, K. V., and S. Goodbourn. 1989. Double-strandedRNA activates binding of NF-kappa-B to an inducible element in the human beta-interferon promoter. EMBO J. 8:1129-1138.

53. Weinberger, J., P. S. Jat, and P. Sharp. 1988. Localization of a repressive sequence contributing to B-cell specificity in the immunoglobulin heavy-chain enhancer. Mol. Cell. Biol. 8:988992.

54. Winoto, A., and D. Baltimore. 1989. $\alpha \beta$ lineage-specific expression of the $\alpha \mathrm{T}$ cell receptor gene by nearby silencers. Cell 59:649-655.

55. Winoto, A., and D. Baltimore. 1989. A novel, inducible and T cell-specific enhancer located at the $3^{\prime}$ end of the $\mathrm{T}$ cell receptor $\alpha$ locus. EMBO J. 8:729-733.

56. Wirth, T., and D. Baltimore. 1988. Nuclear factor NF-kB can interact functionally with its cognate binding site to provide lymphoid-specific promoter function. EMBO J. 7:3109-3113.

57. Yu, H., B. Porton, L. Shen, and L. A. Eckhardt. 1989. Role of the octamer motif in hybrid cell extinction of immunoglobulin gene expression: extinction is dominant in a two enhancer system. Cell 58:441-448. 